\title{
ПРОБЛЕМА НАУЧНОГО ОБЪЯСНЕНИЯ В СОВРЕМЕННОЙ ЗАРУБЕЖНОЙ ПСИХОЛОГИИ
}

\section{В.А. МАЗИЛОВ ${ }^{\mathrm{a}}$ А.А. КОСТРИГИН}

\author{
а Ярославский государственный педагогический университет им. К.Д. Уиинского, 150000, Россия, \\ Ярославль, ул. Республиканская, д. 108/1

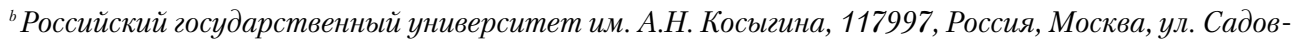 \\ ническая, д. 33
}

\section{Резюме}

В статье обсуждается методологическая проблема научного объяснения в психологии. Отмечено недостаточное освещение данной проблемы в отечественной научной литературе. Авторы обращаются к результатам зарубежных исследований, посвященных проблеме научного объяснения. Выделяются следующие направления исследований в данной области: концептуализация и определение данного понятия, его место в системе методологии науки и психологии, описание случаев применения; различные модели, подходы, схемы объяснения (основанные на средствах и результате; феноменологические, бескомпонентные, механистические и функциональные; редукционизм, механистический анализ, объяснительный плюрализм, теория динамических систем, информационная теория) психологических феноменов, фактов, результатов исследований; методы и техники объяснения (инстанцирование, дедуктивно-номологический метод, метод выведения (наи)лучшего объяснения, реификация, функциональный абстракционизм, функционализация, схематическое доказательство, объяснительная привлекательность, теория объяснительной согласованности); соотношение и сравнение двух ведущих видов объяснения в психологии механистического и функционального, определение их специфики, а также сфер, в которых они наиболее применяемы и эффективны; анализ проблемы объяснения в различных областях психологии (бихевиоризм, эволюционная психология, социальная психология); обсуждение проблемы плюрализма объяснительных моделей в психологии как признание сложности психики и психических явлений. Авторы отмечают, что зарубежные психологические исследования касаются специфичных вопросов теоретических и прикладных проблем, но не занимаются теоретико-методологической перестройкой всей системы психологии. Обоснована важность обсуждения научного объяснения в отношении его потенциала для решения актуальных методологических проблем психологии.

Ключевые слова: методология психологии, психологическое объяснение, научное объяснение, зарубежная психология, модели объяснения, механистическое объяснение, функциональное объяснение, научный метод.

Исследование выполнено в рамках проекта Российского фонда фундаментальных исследований «Объяснение в психологической науке: разработка уровневой концепции» (№ 18-013-00137). 


\section{Введение}

Объяснение относится к числу вечных проблем философии и науки. Собственно, сама философия возникла как попытка античных мыслителей понять и объяснить мир, в котором жил человек. Объяснение уже тогда было значимой ценностью. К ХХ в. были сформулированы основные направления объяснительных практик, была прослежена периодизация развития объяснения в истории науки и философии (Конт, 1910).

Специфика решения проблемы объяснения преломляется в предмете, методе, парадигме и истории развития конкретной науки. В философии науки $\mathrm{XX}$ в. изначально возникла дедуктивно-номологическая модель объяснения, затем появлялись уточняющие позиции, учитывающие тот или иной аспект анализа феноменов или научных данных, - модель статистической релевантности (значимости), каузально-механистическая модель, унификационистская модель (Glennan, 2002; Newton-Smith, 2000; Salmon, 2006; Woodward, 2019). Данные модели обладают рядом достоинств и недостатков, однако все они в той или иной степени использовались в гуманитарных науках, в частности в психологии.

Мы не ставим задачу детального рассмотрения исторического развития проблемы объяснения в психологии, однако отметим, что в Новое время наблюдается триумф причинно-следственного объяснения. Дело в том, что в эту эпоху происходит бурное развитие естественных наук, которые продуктивно опираются именно на этот вид объяснения. Формулируются законы, которые выражаются языком математических формул. Успехи естествознания подтверждают продуктивность причинно-следственного объяснения.

Как ни удивительно, теория объяснения в психологии представляет актуальную проблему. Приходится констатировать, что удовлетворительная теория объяснения в психологии, к сожалению, отсутствует.

В научной психологической литературе полноценные объяснения встречаются крайне редко. В диссертациях, научных монографиях чаще всего речь идет об интерпретации. Обратимся к классической работе Б.Г. Ананьева, в которой дается классификация методов психологического исследования. Б.Г. Ананьевым, как известно, выделяются следующие группы методов: 1) организационные (в эту группу входят сравнительный, лонгитюдинальный, комплексный); 2) эмпирические (в эту группу входят обсервационные, экспериментальные, психодиагностические, праксиметрические и биографические); 3) обработки данных (количественные и качественные методы анализа); 4) интерпретационные методы (различные варианты генетического и структурного методов) (Ананьев, 1996). В этой классификации методов психологического исследования (лучшей пока не предложено) объяснение не предусмотрено. Такую ситуацию можно интерпретировать как неблагополучную.

Вспомним, что такое объяснение. Объяснение представляет собой главную, основную функцию науки. «Слово “объяснение” употребляется в самых различных смыслах и в повседневной жизни, и в научном языке. Среди этих смыслов можно выделить два основных, которые принимались в качестве 
отправных в разработке тех или иных концепций объяснения в эпистемологии и философии науки. Первый смысл связан с представлением о том, что для объяснения некоторого явления необходимо выявить некоторую скрытую за ним “сущность”, “внутреннюю природу”. Такого рода трактовки объяснения были характерны для метафизики, натурфилософии, а также ранних стадий развития науки. По мере развития научного познания такое понимание было вытеснено иным, в котором объяснение предполагает включение явления в структуру некоторых регулярностей, законов, в контекст целостной теории. Эти две общие перспективы могут пересекаться, поскольку возможен такой взгляд на теорию, в котором ее функция видится в раскрытии сущности определенного круга явлений. Однако подобное “эссенциалистское” понимание теории находит ныне все меньше сторонников» (Объяснение в философии науки, 2008, с. 7).

Интерпретация в широком смысле (лат. Interpretatio) - «разъяснение, истолкование» - процедура, которая должна быть направлена на достижение лучшего понимания. Уместно вспомнить, что интерпретация была на протяжении многих веков основным методом в философской психологии (Роговин, 1969; Мазилов, 1998). Это гибкий и достаточно универсальный традиционный психологический метод. Основные этапы развития интерпретации в философской психологии прослежены в книге М.С. Роговина (1969). Как отмечает B.C. Швырев, «в гуманитарном знании, в науках о культуре понятие “интерпретация” употребляется в значении, близком к понятию понимания, в котором, начиная с Дильтея, стремятся выразить специфику гуманитарного и культурологического познания, направленного на постижение (расшифровку, декодирование) смысла, воплощенного в различных текстах и вообще артефактах культуры. В философской герменевтике (Э. Бетти, Х. Гадамер) проблематика интерпретации выходит за рамки постижения смыслов текстов, оказываясь связанной с познанием бытия человека в мире» (Швырев, 2010, c. 134).

В начале XXI в. в отечественной психологии сохраняется актуальность проблемы причинно-следственного объяснения. Попытки его осуществления зачастую приводят в тупик: использование этого вида объяснения ведет к редукции психического к непсихическому. И это отдельная тема. И поскольку объяснение (или его видимость) в психологии все же должно присутствовать, используется интерпретация, под которой чаще всего имеются в виду другие видъ объяснения (не причинно-следственные). Их обыкновенно и именуют интерпретацией в научном исследовании. Во избежание возможных недоразумений скажем, что авторы данной статьи отнюдь не являются противниками причинно-следственного объяснения. Напротив, в специальной статье одного из авторов обсуждается вопрос, как вывести решение проблемы использования причинно-следственного объяснения в психологии из возникшего тупика (Мазилов, 2020).

В отечественной психологии проблеме объяснения не уделялось должного внимания. Философской базой науки служила марксистско-ленинская философия, объяснение было прерогативой материалистической диалектики, 
которая объясняла все, а инструментом объяснения выступали три закона диалектики и система из 16 основных категорий. Отметим, что и в постсоветский период, когда диалектический и исторический материализм утратил статус «единственного верного», теоретических работ по проблеме объяснения в психологии было опубликовано немного (Залевский, 2008; Юревич, 2006, 2008; Мазилов, 2008).

В мировой психологии наиболее разработанной была концепция объяснения, разработанная Ж. Пиаже (Piaget, 1963). В настоящее время эту теорию следует признать существенно устаревшей. Критике теории объяснения Ж. Пиаже были посвящены специальные работы (Мазилов, 2017б, 2018б; Роговин, 1979). В частности, отмечалось, что, согласно Пиаже, следующему картезианской традиции, сознание и поведение разрываются, причинность применяется лишь к поведению: необходимо «допустить существование двух различных рядов явлений, один из которых образован состояниями сознания, а другой - сопровождающими их нервными процессами (причем всякое состояние сознания соответствует такому процессу, а обратное было бы неверно). Связь между членами одного из рядов и членами другого ряда никогда не является причинной связью, а представляет собой их простое соответствие, или, как обычно говорят, “параллелизм”» (Пиаже, 1966, с. 188). Причинное объяснение, применяемое к поведению, неизбежно приводит к редукционизму, т. е. фактически сводит его либо к биологии, либо к социологии: объяснение сводится к редукции, а формы его обусловлены используемым «субстратом».

Здесь нет возможности развивать эту мысль подробнее, отметим лишь, что классик психологии выступает наследником картезианского дуализма, противопоставляя сознание и поведение. Как следствие, Ж. Пиаже приходит к выводу о неизбежности использования в психологии психофизиологического параллелизма, что явно противоречит позициям и традициям отечественной психологии.

Обсуждая проблему объяснения в психологии, нельзя абстрагироваться от другой важнейшей методологической проблемы психологии - проблемы предмета. В зависимости от того, что предполагают предметом в конкретном психологическом исследовании, подбирается и своя модель объяснения этого предмета. Анализ работы Ж. Пиаже (и других исследований по проблеме объяснения) убедительно показывает, что главным камнем преткновения оказывается именно специфика психологии и многомерность ее предмета (Мазилов, 2017a, 2018a, 2020).

Обратимся к наиболее востребованным направлениям зарубежной психологии в связи с осмыслением проблемы научного объяснения. В зарубежной науке в последние два-три десятилетия вопросы объяснения затрагиваются в рамках фундаментальной психологии, а также в отдельных психологических отраслях (однако не во всех). Большинство работ посвящено основам разработки психологического объяснения как научного метода, осмыслению различных объяснительных моделей и подходов, применяемых в психологии, приемов и методик внутри психологического объяснения. Это является естественным, так 
как проблема объяснения является сугубо научной, теоретической и фундаментальной.

В конкретных отраслях объяснение обсуждается в основном применительно к бихевиоризму, эволюционной психологии и социальной психологии. Эти области нами будут рассмотрены исходя из значимости «эмпирического» материала - по количеству работ. Это, безусловно, является ограничением и показывает отсутствие репрезентативности, но, с другой стороны, выражает положение вещей в теории и методологии в зарубежной (мировой) психологии. Также отметим, что отдельно нами не обозначается объяснение в рамках когнитивной психологии. Это связано с тем, что когнитивная психология является доминирующим подходом в зарубежной психологии, имеет статус общепсихологического. И работы, посвященные когнитивной психологии, войдут в обсуждение теоретических аспектов объяснения.

В нашем теоретическом обзоре представлено комплексное рассмотрение проблемы объяснения в зарубежной психологической литературе: начав с работ по фундаментальной и методологической тематике, мы перейдем к конкретным областям (бихевиоризму, эволюционной психологии и социальной психологии). Это важно, поскольку общенаучные подходы и методы всегда локально преломляются и модифицируются в конкретной сфере в зависимости от предмета исследования и доминирующей в конкретный момент методологии.

\section{Теоретические основы концептуализации и применения объяснения как научного метода}

M. Коломбо определяет объяснение как самостоятельный метод (Colombo, 2017). Он считает, что объяснение как научная операция имеет независимое положение и отличается от когнитивной салиентности (cognitive salience понимания значимости какой-либо вещи), рационального признания приемлемости (rational acceptability) и умозаключения (entailment). Объяснение выполняет важные познавательные функции: создает основу для надежных обобщений, способствует обучению и решению задач, играет важную роль в операциях подтверждения и вывода.

Дж. Мур, ссылаясь на М.Б. Тернера (Turner, 1967), считает, что в научном объяснении необходимо выделять две формы: 1) инстанцирование (instantiation) - событие или явление объяснено, когда его особенности можно описать с помощью какой-либо формулы, закона или суждения; 2) дедукция из покрывающего закона (deduction from covering law) или дедуктивно-номологическое объяснение - событие или явление объяснено, когда его описание является выводом из какого-либо закона и определением набора начальных или ограничительных условий; все вместе это представляет собой умозаключение (Moore, 2000). В психологии примером инстанцирования является объяснение психофизического закона Стивенса - взаимоотношение силы ощущения и раздражителя описывается формулой. Дедуктивно-номологической объяснительной моделью в психологии пользуются в большинстве случаев. 
Д. Папино представляет несколько моделей объяснения действий человека в связи с наличием различных убеждений (правдивых или ошибочных). По его мнению, в психологии существует проблема привязки объяснения к двум основаниям: к правдивости и целесообразности (телеологии). Прежде всего, он отмечает следующее положение: «равдивость или ложность убеждения не соответствует его объяснительной роли» (Papineau, 1990, p. 22). Это означает, что убеждение может объяснять действие вне зависимости от того, является ли оно правдивым или ложным; действие человека будет в любом случае объяснено. Д. Папино формулирует две схемы объяснения:

1. Основанную на средствах

a) $X$ хочет $t$

б) $X$ убежден, что s приведет к $t$,

в) $X$ делает $s$.

2. Основанную на результате

a) $X$ хочет $t$,

б) $X$ убежден, что конкретное действие приведет к $t$,

в) Это убеждение правдиво,

г) $X$ достигает $t$.

Преимущества схемы, основанной на средствах, заключается в том, что здесь не вводится дополнительных переменных: с помощью этого средства субъект либо достигнет результата, либо нет. Однако недостатком этой схемы является ограниченность анализа поведения человека и его психических образов и личностных свойств.

Преимуществом объяснительной схемы, основанной на результате, является включение в анализ самих убеждений и целей. Однако присутствует сложность с анализом правдивости убеждений: необходимо вводить дополнительные положения, например, о том, что такое правдивое убеждение «гарантия того, что действие, основанное на этом убеждении, приведет к достижению результата» (Ibid., p. 27) или «убеждение правдиво, если и только если произойдет $p$ » (Ibid., p. 30), как связано правдивое убеждение с достижением результата и удовлетворенностью от него (удовлетворением желания или потребности - «действия, основанные на правдивых убеждениях, удовлетворяют желания/цели, на которые они были направлены» (Ibid., p. 35) или «состояние удовлетворения желания это такое состояние, которое гарантированно случится после действий, соответствующих желанию, если убеждения в основе действий являются правдивыми» (Ibid.) и др.

Д.А. Вейскопф выделяет четыре вида объяснительных моделей: феноменологические, бескомпонентные (noncomponential), механистические и функциональные (Weiskopf, 2011). По его мнению, феноменологические модели не подходят для надежного и прогнозирующего объяснения, только для описания; остальные три модели удовлетворяют критериям научности. Бескомпонентный анализ представляет собой более наукообразное описание психических образов, механистический анализ редуцирует психические явления до механизмов и физиологических процессов, функциональный анализ оперирует когнитивными функциями и процессами как несводимыми к 
физиологическим единицам. Также Д.А. Вейскопф описывает техники, с помощью которых работают данные модели: 1) реификация (reification) овеществление абстрактного понятия (пример: овеществление символических образов через вычислительные системы); 2) функциональный абстракционизм - разложение сложной системы на подсистемы, уровни, слои и др.; 3) функционализация - включение компонентов в систему, которые не соответствуют элементам этой системы, но позволяют системе эффективно функционировать.

М. Марраффа и А. Патерностер считают недостаточными разрозненно существующие объяснительные модели в когнитивных науках: редукционизм, механистический анализ, объяснительный плюрализм, теория динамических систем, информационную теорию и др. (Marraffa, Paternoster, 2013). Они признают, что психические (когнитивные) явления требуют множества и разнообразия объяснительных уровней, которые взаимодействуют между собой через процессы декомпозиции и контекстуализации. Современные достижения в области психологии и когнитивных наук требуют объединения различных теорий и использования плюрализма. По мнению авторов, существующие плюралистические идеи не удовлетворяют всем особенностям психических явлений. М. Марраффа и А. Патерностер предлагают синтез двух подходов - информационного (computationalism) и динамического (dynamicism). Информационный подход полагает, что психика - это вычислительная система, в которой функционирует информация, что выражается в нейронной активности. Динамический подход описывает психику и психические процессы как сложные, нелинейные, самоорганизующиеся, эмерджентные (самовозникающие) системы. Согласно информационно-динамическому подходу, на низших уровнях явления объясняются информационными и механистическими моделями, но на высших они уступают место динамическим: чем сложнее процесс, тем менее он укладывается в механистические, узкие рамки, тем больше он требует к себе нелинейного подхода (Kaplan, Bechtel, 2011). С одной стороны, каждая система имеет части, их изучение осуществляется с использованием информационного подхода, с другой - взаимодействие между частями и системами происходит уже по динамическим закономерностям. Такой подход является интегральным.

Б.Д. Хейг обращается к методу «выведения (наи)лучшего объяснения» (inference to the best explanation, иногда в зарубежной литературе его называют абдукцией, выдвижением гипотез или объяснительной индукцией) как к одному из признанных методов в психологическом объяснении (Haig, 2009). Метод ВНО (выведение (наи)лучшего объяснения) основывается на качественном анализе и рассуждении о научных данных и явлениях. BНО функционирует как метод, который определяет, насколько та или иная теория хорошо объясняет данные, результаты исследований, феномены и др. (Lipton, 2003). Б.Д. Хейг выделяет три подхода в рамках ВНО: схематическое доказательство (schematic argument), объяснительная привлекательность (loveliest explanation) и теория объяснительной согласованности (theory of explanatory coherence). 
Схематическое доказательство представляет собой следующую последовательность рассуждений:

$P 1, P 2 \ldots$ - новые эмпирические феномены и данные,

Теория $T$ объясняется $P 1, P 2 \ldots$,

Ни одна другая теория не может объяснить $P 1, P 2 \ldots$ так же хорошо, как это делает $T$.

Следовательно, $T$ принимается как наилучшее объяснение.

Подход объяснительной привлекательности заключается не столько в вероятности объяснения научных результатов с помощью конкретной теории или гипотезы, сколько в наличии ее объяснительных «достоинств». К последним относятся объединяющая сила, ясность и проработка объяснительных механизмов. Из равных по предсказательной способности теорий выбирается именно та, которая обладает наиболее развитыми перечисленными достоинствами.

Теория объяснительной согласованности сосредоточивается на содержании теорий и гипотез, с помощью которых объясняются результаты. Объяснительная согласованность раскрывается через семь принципов: симметрии, объяснения, аналогии, приоритета эмпирических данных, противоречия, конкурентности, принятия. Чтобы определить степень объяснительной согласованности конкретной теории, необходимо ее рассмотреть на соответствие трем критериям: объяснительной мощности, простоты и аналогии (подобия уже принятым теориям).

Недостатками метода ВНО Б.Д. Хейг считает следующие: 1) выбор теории из уже имеющихся, что не гарантирует высокой степени ее доказательности и истинности; 2) субъективность выбора теории.

М. Колтхарт и М. Дэвис также приходят к выводу о том, что в психологии и когнитивных наук зачастую имплицитно используется метод ВНО (Coltheart, Davies, 2003). Они полемизируют с Дж. Данном и К. Кирснером (Dunn, Kirsner, 2003) относительно того, какие выводы можно делать на основе данных нейропсихологических и когнитивных исследований. Критикуется следующая логическая последовательность (D - данные, T - теория):

D подталкивают к выводу, что T верна,

$\mathrm{D}$ поддерживают вывод, что T верна,

D означают, что T верна,

Если мы наблюдаем D, то Т должна быть верна.

М. Колтхарт и М. Дэвис полагают, что данные не могут быть свидетельством в пользу теорий, тем более призывать, подталкивать или поддерживать их. Данные не могут гарантировать правдивость той или иной теории. Это логическая ошибка. Вывод из данных - это не дедукция и не индукция, а абдукция, т.е. «выведение (наи)лучшего объяснения». Суждение о правдивости теории делается исследователем, а не выводится из данных.

Т. Тео ставит проблему отсутствия признания необходимости опоры на плюрализм в психологическом дискурсе (Тео, 2010). Опираясь на В. Дильтея, он считает, что психология находится на совсем другом онтологическом уровне по сравнению с классической физикой и эволюционной биологией, которые 
используют исторически первые сложившиеся объяснительные модели дедуктивно-номологическую и природно-историческую. Онтологически психические процессы и явления должны изучаться гуманистическими науками (которые представляют собой совсем иную методологию в отличие от естественных наук) с помощью герменевтического метода и с учетом культурноисторического контекста. Особенности психической жизни обусловливают необходимость применения герменевтики для ее постижения. Отсюда вытекает и плюралистическая методология познания психического: мы не знаем наверняка, как именно устроена психика, это приводит к конструированию моделей и понимающей ориентации, что, в свою очередь, создает множество интерпретаций; более того, дополнительными условиями становятся культурно-исторические особенности и познающего, и познаваемого. Плюрализм должен быть признан априорным принципом в психологии.

Однако М. Коломбо считает плюрализм объяснительных моделей проблемой (Colombo, 2017). По самой своей природе объяснение не может выступать как метод, который приводит к однозначному суждению о предмете или идее. В зависимости от различных факторов для объяснения одного и того же явления могут подойти совсем разные посылки и основания. Таким образом, стоит признать плюрализм объяснения, в том числе и в психологии, однако ожидать от этого каких-либо преимуществ нельзя, необходимо учитывать и делать «поправку на плюрализм».

По нашему мнению, в рассмотренных работах можно выделить две основные проблемы: поиск наиболее подходящей объяснительной модели для психологии и обозначение ошибок в объяснении психологических явлений.

Выбор доминирующей объяснительной модели для психологии является нерешенным вопросом до сих пор. Это связано с тем, что психология находится на стыке гуманитарных и естественных наук. Поэтому, как показывают некоторые авторы, в психологии часто прибегают к механистическому объяснению, редукционизму, к опоре на эмпирические данные, стремлению выразить психические функции с помощью формул, схем, алгоритмов и т.п. На другой стороне располагаются идеи герменевтики, описательного подхода, т.е. полный уход от формального, строгого, детерминистского. Есть и взгляды, соответствующие постмодернизму, - стремлению к плюрализму, для которого главной задачей является объяснение (любой ценой, в любом виде). Но наиболее перспективной из предложенных зарубежными авторами является когнитивная объяснительная модель, которая включает информационное объяснение психики через функционирование динамических систем (Marraffa, Paternoster, 2013).

Однако объяснение, хотя и стремится к точности через учет различных принципов, тем не менее содержит множество ошибок, искажений и даже заблуждений. Многие зарубежные авторы отмечают, что необходимо определить роль эмпирических данных в психологических исследованиях (проблема факта), выявить соотношение объективного и субъективного в психических проявлениях человека, а также учитывать субъективные установки и убеждения самих исследователей. Нам известны принципы постнеклассической науки, однако выйти из круга взаимосвязи фактов и интерпретации до сих не 
получается. В любом случае, даже если исследователь принимает какую-либо теорию или модель на основе строгих фактов или расчетов, то на выбор фактов, формул, в конце концов, методологических основ влияет субъективизм выбирающего их ученого. Вероятно, необходимо продолжать обнаруживать ошибки и искажения в психологическом объяснении, так как их наличие в дискурсе не всегда понятно самому субъекту.

\section{Соотношение механистического и функционального объяснения в психологии, когнитивных и нейронауках}

Х.Л. де Йонг рассматривает различные объяснительные подходы в психогенетике (поведенческой генетике) и биопсихологии, которые показывают характер взаимоотношений между психологией и нейронауками (психофизиологией) (de Jong, 2002). Он выделяет следующие подходы: 1) классическая редукция - сведение к более простому, объяснение с помощью общих законов или более фундаментальных теорий; 2) функционализм/автономия - разделение феноменов психических и физиологических (хотя психические базируются на физиологических), они обладают специфическими особенностями, изучаются в соответствующих исследовательских областях, и под них разработан особый терминологический словарь; 3) элиминативизм (редукционизм новой волны - new wave reductionism) - замена психологической терминологии нейронаучной; 4) объяснительный плюрализм - признание подобия и соответствия психических и физиологических процессов, объединение исследовательских теорий и терминологии для объяснения психических и физиологических явлений (например, теория эвристической идентичности heuristic identity theory), их переплетение, анализ проблематики одной науки с помощью теорий другой, и наоборот, коэволюция и развитие обеих исследовательских областей при помощи друг друга. По оценке Х.Л. де Йонга, ни один из подходов не достигает необходимой стратегии объяснения психического и физиологического (нейронаучного). На этом пути перед объяснительными подходами стоит множество проблем, в том числе проблема множественной реализуемости (multiple realizability), супервентности (supervenience), взаимоотношения теорий между собой и др.

Г. Пиччинини и К. Крейвер также поднимают проблему различий разных видов объяснения в психологии и нейронауках, в частности - механистического и функционального (Piccinini, Craver, 2011). Они считают, что функциональный подход не является самостоятельным, его можно свести к механистическому. Авторы называют функциональное объяснение «наброском механистического объяснения» (a sketch of mechanistic explanation - Ibid., p. 308). Свести функциональное к механистическому можно следующим образом: если функциональное объяснение обращается к внутренним состояниям, то существуют состояния внутренних компонентов, идентификацией и анализом которых уже занимается механистическое объяснение. Даже если функциональное объяснение производить через оперирование черными ящиками (в качестве «ящика» представляется какой-либо психический процесс, 
функция, способность или свойство, важен сам факт их осуществления, а не механизмы, по которым они действуют: черный ящик - неизвестно, что внутри, но функционирует; иногда этот подход называется «боксологией» - boxology), то каждый ящик - это структурный компонент системы; система функционирует, потому что действуют такие-то сила и свойство. Это объяснение также является разновидностью механистического. Таким образом, если заполнить структурные компоненты функциональной системы механистическим содержанием, то получится уже механистическая система. Но, по мнению Г. Пиччинини и К. Крейвера, важно не низвести функциональный подход, а наметить пути взаимодействия функционального и механистического объяснения. Авторы полагают, что нужно говорить об интегративном объяснении - соединении функционального и механистического. Тогда изучение психического будет процессом сближения функциональных идей (психология) и механистических (нейронауки).

У. Бехтель обращается к преимуществам механистического подхода в психологическом объяснении (Bechtel, 2009). Он полагает, что в XX в. этот подход слабо развивался, так как он исторически ассоциируется с редукционистским (редукция душевных/психических явлений и функций к физиологическим процессам). Однако, по мнению У. Бехтеля, существует важное отличие механистического объяснения от редукции: редукционистское объяснение основывается только на органах (структурах мозга) и их операциях, а механистический подход учитывает организацию и структуру органов и отделов мозга, а также средовые условия, в которых происходил какой-либо психический (психофизиологический) механизм. Таким образом, механистическое объяснение в психологии заключается в трех направлениях: объяснение психического процесса с помощью функционирования конкретных органов, структур мозга (знания о том, какие механизмы происходят в каждой мозговой структуре, каким закономерностям они подчиняются), объяснение с помощью закономерностей функционирования системы мозга (взаимосвязи различных структур между собой, их влияние друг на друга), учет средовых условий (физические стимулы, обстановка и др.). У. Бехтель называет такое механистическое объяснение интегративным многоуровневым подходом.

Противоположную точку зрения занимает Л.А. Шапиро (Shapiro, 2016). Несмотря на то что зачастую функциональный подход сводится к механистическому, автор утверждает, что мы должны признать функциональное объяснение самостоятельным. Механистическое объяснение психических явлений и процессов базируется на обосновании их причинно-следственной обусловленности органами, мозговыми структурами, в конце концов, базовыми психическими механизмами. Функциональный объяснительный подход частично базируется на механистическом (ведь функции, которыми описывается то или иное явление, реализуются на основе конкретных элементов, органов и т.п.), но по большей части сосредоточивает свое внимание на процессуальном аспекте, не сводя функции к конкретным механизмам и элементам. Л.А. Шапиро видит перспективу признания автономности функционализма в отношении изучения и объяснения когнитивных процессов. 
Эту же идею разделяет и Д. Бэрретт (Barrett, 2014). Он критикует отождествление функционального и механистического объяснений. Автор рассматривает модель рабочей памяти А. Бэддели и Г. Хитча как пример, когда объяснение психических процессов обходилось без описания нейрофизиологических механизмов и достигало уровня полноценной научной теории. Согласно ученым, рабочая память состоит из зрительно-пространственный наброска, фонологической петли, центрального управляющего элемента, эпизодического буфера и других компонентов. Для понимания функционирования психического процесса не требуется раскрытие физиологических механизмов, так как они не проливают свет на психическое субъективное содержание, а лишь приводят к редукции.

К проблемам механистического и функционального объяснения в психологии обращались и другие авторы (Beaty et al., 2014; Gervais, de Jong, 2013; Illari, 2013; Matyja, 2015; Stinson, 2016).

Соотношение механистического и функционального объяснения действительно является важной проблемой, так как в психологии специалисты рассматривают психический процесс чаще всего именно с естественно-научной точки зрения (психофизиология и нейронауки) и субъективной, собственно психологической (обращение к внутреннему миру человека). Попытки объединить данные подходы в зарубежной психологии (интегративный многоуровневый подход (Bechtel, 2009)), хотя и представляются перспективными, тем не менее не учитывают более фундаментальную проблему психологии определение ее предмета. Мы должны признать, что до сих пор отсутствует единый взгляд на предмет психологии, а значит, не определено, что является первичным - мозг или психика (внутренний субъективный мир). В зарубежной психологии был предложен вариант решения этого вопроса - разработка когнитивной психологии, в которой психический процесс рассматривается как информационный; он зависит от функционирования мозговых процессов (как субстрата для хранения и циркуляции информации). В таком случае синтез механистического и функционального объяснения является наиболее подходящим для когнитивной психологии, так как аксиоматически признается наличие этих двух аспектов психического. Тем не менее отсутствие обсуждения предмета психологии в подобных работах вызывает настороженность, ведь когнитивная психология разрабатывалась достаточно давно, а сейчас возникают новые факты и обстоятельства пересмотра традиционных точек зрения на психику.

\section{Проблема объяснения в отдельных областях психологии}

\section{Бихевиоризм}

Выше мы касались того, как сторонник бихевиоризма Дж. Мур определяет ведущие объяснительные модели в психологии - инстанцирование и дедуктивно-номологическое объяснение (Moore, 2000). Однако, по его мнению, эти подходы можно использовать только в необихевиоризме и когнитивной психологии, 
но никак не в классическом бихевиоризме и поведенческом анализе (behavior analysis). Дж. Мур поднимает две проблемы: что такое вообще объяснение и как объясняют явления бихевиористы. Поведенческие аналитики рассматривают объяснение не как научную практику, но как часть поведения человека, конкретно - вербальное поведение. «Объяснительное поведение» является сложным оперантным поведением, т.е. анализ «объяснительного поведения» состоит из выявления стимулов, которые побуждают такое поведение, и подкреплений, которые поддерживали его. С точки зрения бихевиоризма, объяснять - значит осуществлять поведение по схеме «стимул - реакция» со всеми подкрепляющими факторами. Стимулом для объяснительного поведения могут быть воспринятые данные, неразрешенный вопрос, человек, который побуждает к объяснению, и др. Подкреплением той или иной объяснительной стратегии (выбора того или иного аргумента) могут стать манипулирование событием или предметом на основе объяснения, предсказание или интерпретация, разрешение вопроса, упорядочение данных и др. Это процесс осуществления объяснения как поведения. Но в самом бихевиоризме к этому анализу добавляются элементы полноценного оперантного анализа выявление контекста, средовых факторов, а также - одно из ключевых положений - следование прагматизму: полученное объяснение поведения должно быть полезным с прикладной точки зрения.

Другие представители бихевиоризма У. Баум и Дж. Хит противопоставляют бихевиористическое объяснение интенциональному психологическому (Baum, Heath, 1992). Согласно интенциональному объяснению, поведение человека - результат какого-либо психического процесса (например, человек «захотел» что-то сделать). Но такое объяснение, с точки зрения авторов и Б. Скиннера, является «менталистским», психологизированным. Слабое место данного объяснения заключается в том, что оно вымышленное, придуманное (fictional), мы лишь предполагаем, что что-то могло вызвать определенное поведение, наверняка мы не можем знать. Поэтому такой подход бесплодный и не может быть практичным/прагматичным (к чему стремятся бихевиористы). В противовес интенциональному подходу бихевиористы используют физическое объяснение с элементами исторического. Поведенческие аналитики, во-первых, ищут физические причины какого-либо поведения в окружающей среде, а во-вторых, индивидуально-исторически прослеживают, как конкретное действие могло сформироваться. Человек решил какую-то задачу не потому, что захотел, пришел к ней интеллектуально и т.п., но на него повлияли средовые факторы, он в прошлом уже сталкивался с такой задачей или наблюдал, как ее решают другие. Поэтому бихевиоризм в некотором смысле - это «история поведения» (history of behavior). У. Баум и Дж. Хит видят здесь сближение бихевиоризма с эволюционной биологией и психологией.

Хотя классический бихевиоризм является достаточно устаревшим подходом, обсуждение его объяснительных моделей является важным. Во-первых, мы можем проследить развитие объяснения в психологии (в частности, в зарубежной психологии) и понять, по каким причинам бихевиористическое 
объяснение на определенном этапе было доминирующим и до сих остается в американской психологии и почему произошел отказ от него, например, в пользу гуманистического. Во-вторых, бихевиористическое объяснение наиболее соответствует повседневности, а значит, каждый исследователь вне «лабораторных условий», вне эксперимента и исследований пользуется им. Важно понять, как бихевиористическое обыденное объяснение может пересекаться с научным исследовательским и влиять на него. Наконец, рассмотрение объяснительных практик в качестве самостоятельного поведения также открывает нам новые направления исследований: возможно, существование и актуализация определенных условий запускают конкретные объяснительные поведенческие проявления, обстоятельства обусловливают научно-исследовательское поведение, объяснение.

\section{Эволючионная психология}

Д. Червоне обращается к эволюционной психологии и полагает, что ее можно использовать для успешного объяснения в психологии личности (Cervone, 2000). Базовые посылки эволюционной психологии следующие: 1) эволюционная теория; организм стремится к приспособлению к окружающей среде; 2) психика - продукт эволюции, состоит из психических органов (модулей); 3) психические органы являются контекстно-специфичными (обрабатывают сигналы только ограниченного ряда стимулов, вырабатывают решения только на конкретные ситуации); 4) каждый отдельный психический модуль занимается только специфической задачей, а не является многозадачным (как это трактуется, например, сторонниками теории черт); 5) поведение рассматривается как взаимоотношение психических механизмов и средовых сигналов; 6) поведение является приспособлением к окружающей среде, поиску ресурсов, т.е. современным выражением поведения на предыдущих стадиях эволюции. Однако существуют и проблемы: пока неизвестно, как формируются психические модули, к каким конкретным ситуациям и стимулам они привязаны, как они выбираются среди аналогичных, является ли мультимодульным сложное поведение человека и др. Автор считает, что некоторые из этих положений реализуются (или заимствованы) в других подходах психологии личности: в когнитивной психологии - модульная организация психики; в социальном бихевиоризме - контекстно-специфическое поведение и др.

Д.М. Басс выделяет несколько эволюционно-психологических концептов, с помощью которых можно интерпретировать личностные свойства и индивидуальный путь личности (Buss, 2009): 1) согласно теории жизненной истории (life-history theory), человеческая жизнь, ее стратегии и сценарии строятся на основе решений относительно того, в какое русло будет направлять свою биологическую энергию человек, каким образом он будет адаптироваться и приспосабливаться к биологическим условиям, реализуя человеческий, социальный, интеллектуальный и другие потенциалы; 2) теория высокозатратных сигналов (costly signaling theory) - рассмотрение человека как социальное 
животное, которое отправляет сигналы-запросы на получение чего-либо важного для себя, но которое предлагает взамен тоже что-то ценное (например, часто среди иллюстраций такого поведения приводят просоциальное, альтруизм и т.п.); 3) балансный отбор (balancing selection) реализуется через два механизма: средовая гетерогенность (environmental heterogeneity) (требует от определенной личности конкретного набора свойств или качеств) и частотнозависимый отбор (frequency-dependent selection): наиболее приспособляемой и успешной будет та личность, которая обладает несколькими стратегиями поведения и др. Эволюционно-психологическое объяснение используется даже в прикладных сферах, например, в маркетинге и микроэкономике (Prendergast, Ching, 2013).

Р.Д. Грей, М. Хини и С. Фэйрхол критикуют эволюционно-психологическое объяснение за преувеличение адаптационного принципа, отсутствие анализа лежащих в основе адаптационного поведения когнитивных и нейромеханизмов, модулярную структуру психики и концепцию мономорфической психики (monomorphic mind) (Gray et al., 2003).

Эволюционная методология и объяснительная модель вводят в психологию измерение среды и социума, таким образом, объяснение психических явлений усложняется, так как они встраиваются в более разнообразную и многофакторную систему. С одной стороны, благодаря этому мы приближаемся к пониманию человеческого поведения и психических процессов, с другой - наоборот, теряем шансы объяснить что-либо из-за того, что все учесть не получится. Поэтому эволюционно-психологическая точка зрения имеет множество безусловных принципов и положений. В частности, концепт «жизненной истории» (Buss, 2009) соединяет биологическое, психическое и социальное и объясняет человеческую жизнь через разнонаправленные действия и решения, однако все это является теоретическим конструктом. Как нам представляется, эволюционно-психологическое объяснение может быть перспективным, если сделать попытку осмысления фундаментальной методологии психологии: например, ответить на вопросы, насколько организменными являются психические проявления, насколько они подчиняются эволюционным законам и т.п.

\section{Социальная психология}

А. Месуди намечает связь между эволюционной психологией и социальной психологией, полагая, что можно либо организовать новую научно-исследовательскую область, либо укреплять междисциплинарные связи между данными науками (Mesoudi, 2009). По его мнению, эволюционно-психологическая модель объяснения как минимум может быть полезна для социальнопсихологической интерпретации, а как максимум - может преобразовать уже сложившиеся классические социально-психологические теории. Так, на примере пяти феноменов он показывает, как перекликаются объяснительные модели этих двух наук:

1) социальное научение: 
а) социальная психология - приобретение социального и культурного опыта;

б) эволюционная психология - с помощью наблюдения за поведением других человек меньше затрачивает своих ресурсов и энергии;

2) конформность:

а) подчинение большинству, социальноодобряемое поведение;

б) приобретение качества или мнения, которое наиболее популярно или распространено в популяции;

3) установка на следование успешному социальному примеру (социальное подражание) (model-based bias/prestige-success bias):

a) следование социальному примеру, так как он наиболее успешен и предпочтителен;

б) следование наиболее адаптивному примеру;

4) апперцепция (content bias):

a) запоминание и понимание только знакомого;

б) генетическая предрасположенность к усвоению какой-либо информации;

5) межгрупповые процессы:

а) социальная идентичность («мы» и «они»);

б) эволюционно более сплоченные группы сильнее несплоченных, тенденция к поддержке своей группы, так как она обеспечивает тебя ресурсами и способствует адаптации.

А. Месуди считает, что социальная психология и эволюционная психология должны сотрудничать и обмениваться теоретическими концептами для более успешной интерпретации и понимания научных проблем.

О. Клен определяет глубинные основы и факторы научного объяснения в социальной психологии (Klein, 2009). По его мнению, теории в социальной психологии на протяжении XX в. во многом зависели от политических установок. Как мы знаем, в социальной психологии есть два большийх вектора индивидуальный и групповой. В зависимости от политической ситуации в обществе развивались индивидуалистические (либерализм) или групповые/коллективистские (социализм и т.п.) идеи. Более того, этот фактор влиял и на постановку социально-психологических проблем и на их объяснение. В XXI в. мы столкнулись с повсеместным распространением нейронаук: появилась и социальная нейронаука (social neuroscience), которая, вопервых, постулирует, что человек - это социальное существо (а не изолированный индивидуум), а во-вторых, изучает социально-психологические феномены и ищет их нейронные и физиологические корреляты и основания вместо индивидуально-психологических. Это новая политическая идея в науке. Автор призывает заниматься «интроспекцией», рефлексией своих научных убеждений и установок и быть объективными и в постановке научных проблем, и в их решении и объяснении.

Как видно на примере работ по социальной психологии, объяснение здесь зачастую касается частных случаев, конкретных феноменов (научение, конформность, подражание и др.), что отдаленно связано с общими явлениями 
этой области. Вероятно, это достаточно характерно в целом для социальнопсихологического знания. Более того, намечается усложнение ситуации за счет создания нового исследовательского поля - социальной нейронауки. Не претендуя на истину, отметим, что пока мало обнаружено нейронных коррелятов для сложных индивидуальных психических феноменов, а социальнопсихологические феномены являются еще более сложными и не локализованными в одном субстрате. К тому же, как отмечают некоторые авторы (Ibid.), социальная психология еще и подвержена политическим и - шире - общественным влияниям на теоретическую и исследовательскую проблематику (хотя, скорее всего, такие влияния можно обнаружить и в других психологических дисциплинах). Можно заключить, что, как и в других проанализированных нами направлениях зарубежной психологии, социальной психологии необходимо поставить задачи рефлексии собственной методологии и объяснительных схем.

\section{Заключение}

Современная зарубежная психология и смежные научные области (когнитивные науки, нейронауки и биопсихология) акцентируют внимание на проблеме научного объяснения психологических данных. Однако анализ проблемы сосредоточивается не на основаниях объяснения как специфичной методологии или направлении теоретических исследований, чем занимались крупные теоретики и методологи психологии в XX в. (Cummins, 1983; Dodwell, 1960; Fodor, 1968; Hutten, 1956). Зарубежные ученые обращаются к конкретным вопросам психологического объяснения:

- объяснительные модели (основанные на средствах и результате; феноменологические, бескомпонентные, механистические и функциональные; редукционизм, механистический анализ, объяснительный плюрализм, теория динамических систем, информационная теория);

- методы и техники объяснения (инстанцирование, дедуктивно-номологический метод, метод выведения (наи)лучшего объяснения; реификация, функциональный абстракционизм, функционализация, схематическое доказательство, объяснительная привлекательность, теория объяснительной согласованности);

- проблема плюрализма объяснительных моделей в психологии как признание сложности психики и психических явлений;

- соотношение механистического и функционального объяснения в психологии, когнитивных и нейронауках как элемент доминирующей парадигмы в зарубежной науке;

- проблемы объяснения в отдельных областях психологии: бихевиоризм (оперантный анализ объяснительного поведения и история поведения); эволюционная психология (возможность применения эволюционно-психологических объяснений к личности и индивидуальным различиям - теория жизненной истории, теория высокозатратных сигналов, балансный отбор), социальная психология (междисциплинарное взаимодействие социальной 
психологии и эволюционной психологии, факторы социально-психологического объяснения - политика, общественный запрос и нейронауки).

Как видно, в основном зарубежные психологические исследования касаются специфичных вопросов теоретических и прикладных проблем, не сосредоточены на теоретико-методологической перестройке всей системы психологии. Мы должны констатировать, что доминирующими парадигмами в зарубежной психологии до сих пор остаются бихевиоризм и когнитивные науки, добавляются к ним нейронауки. Не намечается выхода из ограниченности и многообразия психологических теорий психики и личности, а лишь решаются ситуационные проблемы. Тем не менее такая изолированность объяснительных моделей в бихевиоризме, когнитивных науках и нейронауках не будет обнаруживать проблем в объяснении и понимании психического и личностного, если будет оставаться в рамках своей области.

Но чрезмерное увлечение когнитивными и нейронауками и подмена ими предмета психологии отмечена и некоторым нашими современникам. М.С. Газзанига размышляет о проблемах современной психологии и нейронаук и пытается представить, как инопланетянин, решивший посетить Землю, наблюдал бы за учеными, изучающими мозг и психику (Gazzaniga, 2010). Инопланетянин видит, что представители нейронаук и психофизиологии умеют измерять все психофизиологические показатели с любого органа, в том числе и мозга, могут добираться в своем теоретическом и эмпирическом анализе до самой низшей микрочастицы в мозге, сосчитать все нейроны, составляют различные системы органов и структур, пытаются понять целое через его части. Однако еще философы XIX в. старались показать, что целое познается не в сумме его элементов, а через выделение и анализ качественно нового образования. Улетая, инопланетянин бы сказал: «Земляне погрязли в трясине. Они не видят, что мозг - это устройство для принятия решений, и он должен быть понят именно в этих терминах с этого уровня описания, никак не ниже. Они только частично эволюционировали. Пройдет еще вечность перед тем, как они найдут нас. И возможно, еще вечность им потребуется, чтобы понять себя» (Ibid., p. 292). И мы добавим: такое положение вещей присутствует не только в нейронауках, но и в самой психологии (ее гуманитарной направленности). Множество редукций или, наоборот, усложнений, эклектизм, «изобретение велосипеда» и т.п. наблюдается сегодня в психологии.

Подводя итоги нашего обзора, можно сделать несколько выводов. С момента публикации упоминавшейся в начале статьи работы Ж. Пиаже прошло более полувека. Констатируем, что общей теории объяснения так и не предложено. Как представляется, причина понятна. Можно предположить, что отсутствие такой теории не в последнюю очередь связано с невниманием зарубежных исследователей к общим вопросам методологии и, в первую очередь, к проблеме предмета психологической науки. В зарубежной психологии отсутствует широкая трактовка предмета как совокупного, позволяющего перенести объяснение (в том числе и причинно-следственное) внутрь предмета. Предложенные объяснительные модели при таком узком подходе неизбежно приобретают характер частных. Попытки отразить реальную 
сложность объяснения и неудовлетворенность частными моделями ни к чему, кроме эклектического соединения элементов различных объяснительных моделей, привести не может. Как нам представляется, большие перспективы на успех имеют подходы к объяснению, исходящие из общей трактовки предмета психологии и переносящие объяснение внутрь предмета, трактуемого как внутренний мир человека (Мазилов, 2020).

\section{Литература}

Ананьев, Б. Г. (1996). Психология и проблемы человекознания. М./Воронеж: Институт практической психологии $/ \mathrm{HПО} \mathrm{«МОДЭК».}$

Залевский, Г. В. (2008). Объяснение и понимание против «циклопной» психологии. Методология и история психологии, 3(1), 41-47.

Конт, О. (1910). Дух позитивной философии. СПб.: Вестник Знания.

Мазилов, В. А. (1998). Теория и метод в психологии. Ярославль: МАПН.

Мазилов, В. А. (2008). Научная психология: проблема объяснения. Методология и история психологии, 3(1), 58-73.

Мазилов, В. А. (2017, а). Методология психологической науки: история и современность. Ярославль: Ярославский государственный педагогический университет им. К.Д. Ушинского.

Мазилов, В. А. $(2017$, б). Объяснение как функция психологической науки. Ярославский педагогический вестник, 2, 175-186.

Мазилов, В. А. (2018, а). Научный факт в психологии: структурно-уровневый подход. Вестник Российского фонда фундаментальных исследований. Гуманитарные и общественные науки, 1, 133-142.

Мазилов, В. А. (2018, б). Разработка концепции объяснения в психологии. Ярославский педагогический вестник, 4, 188-197.

Мазилов, В. А. (2020). Предмет психологической науки и проблема объяснения в психологии. Статья первая: трудности объяснения. Высшее образование сегодня, 6, 69-76.

Объяснение в философии науки. (2008). Методология и история психологии, 3(1), 7-8.

Пиаже, Ж. (1966). Характер объяснения в психологии и психофизиологический параллелизм. В кн. П. Фресс, Ж. Пиаже (ред.), Экспериментальная психология (вып. 1-2, с. 157-194). М.: Прогресс. Роговин, М. С. (1969). Введение в психологию. М.: Высшая школа.

Роговин, М. С. (1979). Психологическое исследование. Ярославль: ЯрГУ.

Швырев, В. С. (2010). Интерпретация. В кн. Новая философская энииклопедия (т. 2, с. 134-135). М.: Мысль.

Юревич, А. В. (2006). Объяснение в психологии. Психологический журнал, 1, 97-106.

Юревич, А. В. (2008). Объяснение в психологии. Методология и история психологии, 3(1), 74-87.

Ссылки на зарубежные источники см. в разделе References после англоязычного блока.

Мазилов Владимир Александрович - заведующий кафедрой, кафедра общей и социальной психологии, Ярославский государственный педагогический университет им. К.Д. Ушинского, доктор психологических наук, профессор.

Сфера научных интересов: методология психологии, история психологии, психология способностей, психология образования.

Контакты: v.mazilov@yspu.org 
Костригин Артем Андреевич - старший преподаватель, кафедра психологии, Российский государственный университет им. А.Н. Косыгина (Технологии. Дизайн. Искусство).

Сфера научных интересов: история психологии, наукометрические и цифровые исследования психологии, социальная психология.

Контакты: artdzen@gmail.com

\title{
The Problem of Scientific Explanation in Modern Foreign Psychology
}

\author{
V.A. Mazilov ${ }^{\mathrm{a}}$, A.A. Kostrigin ${ }^{\mathrm{b}}$ \\ ${ }^{a}$ Yaroslavl State Pedagogical University named after K.D. Ushinsky, 108/1 Respublikanskaya Str., \\ Yaroslavl, 150000, Russian Federation \\ ${ }^{b}$ Kosygin Russian State University, 33 Sadovnicheskaya str., Moscoze, 117997, Russian Federation
}

\begin{abstract}
The article discusses the methodological problems of psychology, in particular, the problem of scientific explanation. Insufficient coverage of this problem in the Russian scientific literature is noted. The authors turn to the results of foreign studies, devoted to the problem of scientific explanation. The following areas of research in this field are determined: conceptualization and definition of this concept, its place in the system of methodology of science and psychology, description of application cases; various models, approaches, schemes of explanation (based on means and result; phenomenological, componentless, mechanistic and functional; reductionism, mechanistic analysis, explanatory pluralism, theory of dynamical systems, information theory) of psychological phenomena, facts, research results; methods and techniques of explanation (instantiation, deductive-nomological method, inference to the best explanation, reification, functional abstractionism, functionalization, schematic proof, explanatory appeal, theory of explanatory consistency); correlation and comparison of the two leading types of explanations in psychology - mechanistic and functional, determining their specificity, as well as the areas in which they are most applicable and effective; analysis of the problem of explanation in various fields of psychology (behaviorism, evolutionary psychology, social psychology); discussion of the problem of pluralism of explanatory models in psychology as an acknowledgment of the complexity of the mind and mental phenomena. The authors note that foreign psychological research deals with specific issues of theoretical and applied problems, but does not engage in theoretical and methodological restructuring of the entire system of psychology. The importance of discussing scientific explanation is grounded in relation to its potential for solving urgent methodological problems of psychology.
\end{abstract}

Keywords: methodology of psychology, psychological explanation, scientific explanation, foreign psychology, explanation models, mechanistic explanation, functional explanation, scientific method. 


\section{References}

Ananiev, B. G. (1996). Psikhologiya i problemy chelovekoznaniya [Psychology and problems of human knowledge]. Moscow/Voronezh: Institute of Practical Psychology/NPO "MODEK". (in Russian)

Barrett, D. (2014). Functional analysis and mechanistic explanation. Synthese, 191(12), 2695-2714. doi:10.1007/s11229-014-0410-9

Baum, W. M., \& Heath, J. L. (1992). Behavioral explanations and intentional explanations in psychology. American Psychologist, 47(11), 1312-1317. doi:10.1037/0003-066X.47.11.1312

Beaty, R. E., Benedek, M., Wilkins, R. W., Jauk, E., Fink, A., Silvia, P. J., ... Neubauer, A. C. (2014). Creativity and the default network: A functional connectivity analysis of the creative brain at rest. Neuropsychologia, 64, 92-98. doi:10.1016/j.neuropsychologia.2014.09.019

Bechtel, W. (2009). Looking down, around, and up: Mechanistic explanation in psychology. Philosophical Psychology, 22(5), 543-564. doi:10.1080/09515080903238948

Brown, R. (1963). Explanation in social science. Chicago, IL: Aldine.

Buss, D. M. (2009). How can evolutionary psychology successfully explain personality and individual differences? Perspectives on Psychological Science, 4(4), 359-366. doi:10.1111/j.17456924.2009.01138.x

Cervone, D. (2000). Evolutionary psychology and explanation in personality psychology: How do we know which module to invoke? American Behavioral Scientist, 43(6), 1001-1014. doi:10.1177/00027640021955720

Colombo, M. (2017). Experimental philosophy of explanation rising: The case for a plurality of concepts of explanation. Cognitive Science, 41(2), 503-517. doi:10.1111/cogs.12340

Coltheart, M., \& Davies, M. (2003). Inference and explanation in cognitive neuropsychology. Cortex, 39(1), 188-191. doi:10.1016/S0010-9452(08)70099-6

Comte, A. (1910). Dukh pozitionoi philosophii [The spirit of positive philosophy]. Saint Petersburg: Vestnik Znaniya. (in Russian)

Cummins, R. (1983). The nature of psychological explanation. Cambridge, MA: A Bradford Book/ The MIT Press.

De Jong, H. L. (2002). Levels of explanation in biological psychology. Philosophical Psychology, 15(4), 441-462. doi:10.1080/0951508021000042003

Dodwell, P. C. (1960). Causes of behaviour and explanation in psychology. Mind. New Series, 69(273), 1-13.

Dunn, J. C., \& Kirsner, K. (2003). What can we infer from double dissociations? Cortex, 39(1), 1-7. doi:10.1016/S0010-9452(08)70070-4

Fodor, J. A. (1968). Psychological explanation. An introduction to the philosophy of psychology. New York: Random House Inc.

Gazzaniga, M. S. (2010). Neuroscience and the correct level of explanation for understanding mind: An extraterrestrial roams through some neuroscience laboratories and concludes earthlings are not grasping how best to understand the mind-brain interface. Trends in Cognitive Sciences, 14(7), 291-292. doi:10.1016/j.tics.2010.04.005

Gervais, R., \& de Jong, H. L. (2013). The status of functional explanation in psychology: Reduction and mechanistic explanation. Theory and Psychology, 23(2), 145-163. doi:10.1177/ 0959354312453093

Glennan, S. (2002). Rethinking mechanistic explanation. Philosophy of Science, 69(3), S342-S353. doi:10.1086/341857 
Gray, R. D., Heaney, M., \& Fairhall, S. (2003). Evolutionary psychology and the challenge of adaptive explanation. In K. Sterelny \& J. Fitness (Eds.), From mating to mentality: Evaluating evolutionary psychology (pp. 247-268). London/New York: Psychology Press.

Haig, B. D. (2009). Inference to the best explanation: A neglected approach to theory appraisal in psychology. The American Journal of Psychology, 12(2), 219-234.

Hutten, E. H. (1956). On explanation in psychology and in physics. The British Journal for the Philosophy of Science, 7(25), 73-85.

Illari, P. (2013). Mechanistic explanation: Integrating the ontic and epistemic. Erkenntnis, 78(2), 237-255. doi:10.1007/s10670-013-9511-y

Kaplan, D. M., \& Bechtel W. (2011). Dynamical models: An alternative or complement to mechanistic explanations? Topics in Cognitive Science, 3(2), 438-444. doi:10.1111/j.1756-8765.2011.01147.x

Klein, O. (2009). From Utopia to Dystopia: Levels of explanation and the politics of social psychology. Psychologica Belgica, 49(2-3), 85-100. doi:10.5334/pb-49-2-3-85

Lipton, P. (2003). Inference to the best explanation. London/New York: Routledge.

Marraffa, M., \& Paternoster A. (2013). Functions, levels, and mechanisms: Explanation in cognitive science and its problems. Theory and Psychology, 23(1), 22-45. doi:10.1177/0959354312451958

Materialisty Drevnei Gretsii [Materialists of Ancient Greece]. (1955). Moscow: Gospolitizdat. (in Russian)

Matyja, J. R. (2015). The next step: mirror neurons, music, and mechanistic explanation. Frontiers in Psychology, 6, 409. doi:10.3389/fpsyg.2015.00409

Mazilov, V. A. (1998). Teoriya i metod v psikhologii [Theory and method in psychology]. Yaroslavl: MAPN. (in Russian)

Mazilov, V. A. (2008). Scientific psychology: the problem of explanation. Metodologiya i Istoriya Psikhologii [Methodology and History of Psychology], 3(1), 58-73. (in Russian)

Mazilov, V. A. (2017, a). Metodologiya psikhologicheskoi nauki: istoriya I soveremennost' [Methodology of psychological science: history and modernity]. Yaroslavl': Yaroslavskii gosudarstvennyi pedagogicheskii universitet im. K.D. Ushinskogo. (in Russian)

Mazilov, V. A. (2017, b). Explanation as a function of psychological science. Yaroslavskii Pedagogicheskii Vestnik, 2, 175-186. (in Russian)

Mazilov, V. A. (2018, a). The scientific fact in psychology: a structural-and-level approach. Vestnik Rossiiskogo Fonda Fundamental'nykh Issledovanii. Gumanitarnye i Obshchestvennye Nauki, 1, 133-142. (in Russian)

Mazilov, V. A. (2018, b). Development of the concept of explanation in psychology. Yaroslavskii Pedagogicheskii Vestnik, 4, 188-197. (in Russian)

Mazilov, V. A. (2020). Predmet psikhologicheskoi nauki i problema ob”yasneniya v psikhologii. Stat'ya pervaya: trudnosti ob"yasneniya [The subject of psychological science and the problem of explanation in psychology. First paper: difficulties of explanation]. Vysshee Obrazovanie Segodnya, 6, 69-76. (in Russian)

Mesoudi, A. (2009). How cultural evolutionary theory can inform social psychology and vice versa. Psychological Review, 116(4), 929-952. doi:10.1037/a0017062

Moore, J. (2000). Varieties of scientific explanation. The Behavior Analyst, 23(2), 173-190. doi:10.1007/BF03392009

Newton-Smith, W. H. (2000). Explanation. In W. H. Newton-Smith (Ed.), A companion to the philosophy of science (pp. 127-133). Malden, MA: Blackwell Publishers. doi:10.1002/ 9781405164481.ch19 
Ob"yasnenie v philosophii nauki [Explanation in the philosophy of science]. (2008). Metodologiya $i$ Istoriya Psikhologii [Methodology and History of Psychology], 3(1), 7-8. (in Russian)

Papineau, D. (1990). Explanation in psychology: Truth and teleology. Royal Institute of Philosophy Supplements, 27, 21-43. doi:10.1017/S1358246100005026

Piaget, J. (1963). L'explication en psychologie et le parallélisme psychophysiologique [Explanation in psychology and psychophysiological parallelism]. In P. Fraisse, J. Piaget \& M. Reuchlin (Eds.), Traité de psychologie expérimentale: Vol. 1. Histoire et méthode [History and method] (pp. 121152). Paris: Presses universitaires de France. (in French)

Piaget, J. (1966). Charakter ob"yasneniya v psikhologii i psikhophisiologicheskii parallelism [Explanation in psychology and psychophysiological parallelism]. In P. Fraisse \& J. Piaget (Eds.), Eksperimental'naya psikhologiya [Experimental psychology] (Iss. 1-2, pp. 157-194). Moscow: Progress. (in Russian)

Piccinini, G., \& Craver, C. (2011). Integrating psychology and neuroscience: Functional analyses as mechanism sketches. Synthese, 183(3), 283-311. doi:10.1007/s11229-011-9898-4

Prendergast, G., \& Ching, L. C. (2013). An evolutionary explanation for shopping behavior.Journal of Consumer Marketing, 30(4), 366-370. doi:10.1108/JCM-02-2013-0456

Rogovin, M. S. (1969). Vvedenie v psikhologiyu [Introduction to psychology]. Moscow: Vysshaya shkola. (in Russian)

Rogovin, M. S. (1979). Psikhologisheskoe issledovanie [Psychological research]. Yaroslavl: YarGU. (in Russian)

Salmon, W. C. (2006). Four decades of scientific explanation. Pittsburgh, PA: University of Pittsburgh Press.

Shapiro, L. A. (2016). Mechanism or bust? Explanation in psychology. The British Journal for the Philosophy of Science, 68(4), 1037-1059. doi:10.1093/bjps/axv062

Shvyrev, V. S. (2010). Interpretatsiya [Interpretation]. In Novaya philosophskaya entsiklopediya [New philosophical encyclopedia] (Vol. 2, pp. 134-135). Moscow: Mysl'. (in Russian)

Stinson, C. (2016). Mechanisms in psychology: ripping nature at its seams. Synthese, 193(5), 15851614. doi:10.1007/s11229-015-0871-5

Teo, T. (2010). Ontology and scientific explanation: Pluralism as an a priori condition of psychology. New Ideas in Psychology, 28(2), 235-243. doi:10.1016/j.newideapsych.2009.09.017

Turner, M. B. (1967). Philosophy and the science of behavior. New York: Appleton-Century-Crofts.

Weiskopf, D. A. (2011). Models and mechanisms in psychological explanation. Synthese, 183(3), 313338. doi:10.1007/s11229-011-9958-9

Woodward, J. (2019). Scientific explanation. In E. N. Zalta (Ed.), The Stanford encyclopedia of philosophy (Winter 2019 Edition). Retrieved from https://plato.stanford.edu/archives/win2019/ entries/scientific-explanation/

Yurevich, A. V. (2006). Explanation in psychology. Psikhologicheskii Zhurnal, 27(1), 97-106. (in Russian)

Yurevich, A. V. (2008). Explanation in psychology. Metodologiya i Istoriya Psikhologii [Methodology and History of Psychology], 3(1), 74-87. (in Russian)

Zalevskii, G. V. (2008). Ob”yasnenie i ponimanie protiv "tsiklopnoi psikhologii” [Explanation and understanding against the "cyclopean" psychology]. Metodologiya i Istoriya Psikhologii [Methodology and History of Psychology], 3(1), 41-47. (in Russian) 
Vladimir A. Mazilov - Head of General and Social Psychology Department, Yaroslavl State Pedagogical University named after K.D. Ushinsky, DSc in Psychology, Professor.

Research Area: methodology of psychology, history of psychology, psychology of abilities, psychology of education.

E-mail: v.mazilov@yspu.org

Artem A. Kostrigin - Senior Lecturer, Psychology Department, Kosygin Russian State University (Technology. Design. Art).

Research Area: history of psychology, scientometric and digital studies in psychology, social psychology.

E-mail: artdzen@gmail.com 